\title{
Transatlantica
}

Revue d'études américaines. American Studies Journal

\section{« Passeurs de la littérature américaine en France}

\section{7-1967 »}

\section{Université Rennes 2}

14-15 mars 2019

\section{Christelle Centi}

\section{(2) OpenEdition}

\section{Journals}

Édition électronique

URL : https://journals.openedition.org/transatlantica/11477

DOI : $10.4000 /$ transatlantica. 11477

ISSN : 1765-2766

Éditeur

Association française d'Etudes Américaines (AFEA)

Référence électronique

Christelle Centi, « « Passeurs de la littérature américaine en France 1917-1967 » », Transatlantica [En ligne], 1 | 2018, mis en ligne le 25 septembre 2019, consulté le 02 février 2023. URL : http:// journals.openedition.org/transatlantica/11477 ; DOI : https://doi.org/10.4000/transatlantica. 11477

Ce document a été généré automatiquement le 2 février 2023.

Creative Commons - Attribution - Pas d'Utilisation Commerciale - Pas de Modification 4.0 International - CC BY-NC-ND 4.0

https://creativecommons.org/licenses/by-nc-nd/4.0/ 


\section{« Passeurs de la littérature américaine en France 1917-1967 »}

Université Rennes 2

14-15 mars 2019

Christelle Centi

1 Les journées d'études "Passeurs de la littérature américaine en France 1917-1967 ", organisées par Benoit Tadié, Anne Reynes-Delobel et Sylvie Bauer dans le cadre d'une collaboration entre l'université d'Aix-Marseille, l'université Paris Nanterre, l'Institut des Amériques de Rennes, les unités de recherche LERMA (EA 853), Centre de Recherches Anglophones CREA (EA 370) et Anglophonie Communautés Ecritures ACE (EA 1796), se sont déroulées les 14 et 15 mars 2019 à l'université Rennes 2. Les échanges portaient sur les personnes ou institutions ayant contribué à la diffusion, la popularisation et la critique de la littérature américaine en France de la fin de la Première Guerre mondiale à la fin des années soixante. La première journée se composait de trois ateliers centrés sur le passage du texte et du contenu: les traducteurs et universitaires, les adaptations et réappropriations, et les poètes; dans sa conférence plénière, Marc Chénetier, en tant que « découvreur » de littérature, s'est attaché à montrer les limites du terme de «passeur ». La deuxième journée, grâce à deux ateliers centrés sur les éléments concrets de la diffusion qu'étaient tout d'abord les lieux de sociabilité (atelier 4) puis la presse, les agents et les imprimeurs (atelier 5), a donné l'occasion à Dominique Jeannerod de présenter en conférence plénière son projet d'analyse quantitative des revues et collections françaises comme vecteurs de diffusion de la littérature policière américaine.

2 Le premier atelier a permis, à travers les figures de Jean Catel comme découvreur et auteur, d'Edith Wharton comme traductrice et de Jack London comme enjeu de traduction, de prendre la mesure des figures littéraires comme à la fois sujets, acteurs, et objets du mouvement de passage de la littérature et culture des États-Unis, dans une dynamique de simplification et de remise en question permanentes.

3 Anne Reynes a offert, à travers des archives inédites portant sur Jean Catel, une analyse du carrefour d'un réseau culturel États-Unis/France, de ses acteurs, des effets 
de cette relation et des facteurs qui l'orientaient. En tant qu'universitaire, traducteur, spécialiste de Whitman et contributeur au Mercure de France, Catel, médiateur culturel, a participé aux reconfigurations, par les périodiques, des échanges transatlantiques et de la géographie littéraire des années 20. Ces reconfigurations ont permis une dynamique d'assignation des identités culturelles nationales, de canonisation, et d'institutionnalisation d'une certaine littérature américaine, dans un contexte peu favorable puisque les écrivains français, à cette époque, se trouvaient davantage préoccupés par la littérature de masse des États-Unis ou par les récupérations idéalisantes ou idéologisantes qu'il était possible d'en faire (à l'exception d'écrivains comme Cocteau et Soupault).

4 Virginia Ricard, de l'université Bordeaux-Montaigne, s'est intéressée à Edith Wharton comme médiatrice de la culture américaine, contre et avec ses stéréotypes. En tant qu'autrice ayant vécu en France dès 1908 et jusqu'à sa mort, Wharton a joué un rôle ambivalent : tout en peignant à travers House of Mirth un portrait peu flatteur de ses compatriotes (obsédés par l'argent, corrompus), elle a dénoncé, dans un discours nouvellement publié, «France and Its Allies at War », donné le 8 février 1918 (devant un public composé notamment de René Viviani, d'ambassadeurs, d'ecclésiastiques et d'écrivains), la facilité à laquelle les Français cèdent parfois dans leur compréhension des États-Unis et les stéréotypes qu'ils perpétuent.

Quant à Jack London, Véronique Béghain, de l'université Bordeaux-Montaigne, a présenté les temporalités d'édition et de traduction de l'auteur, ainsi que la nature kaléidoscopique de son lectorat. Le " passage " de London en France a été l'œuvre de Louis Postif et Paul Guyer, qui ont effectué un travail d'acclimatation (par allègement et clarification d'une langue qu'ils considéraient trop violente et émotive) autant que de diffusion auprès du public français, et ont détenu les droits exclusifs pendant une période d'une longueur exceptionnelle. La figure de Jack London est donc montrée par Véronique Béghain comme une construction conditionnée par notre perception de ce qui est "américain" par rapport à un style purement français, rétroactivement mythologisé, ce qui souligne le travail de filtre des « passeurs » déjà évoqué pour Edith Wharton.

6 La discussion s'est alors dirigée vers cette compétition entre France et Amérique, entre constructions d'un canon «passéiste » et d'une « immaturité » moderne. L'importation des clichés parfois nés localement opère un transfert d'images déjà erronées et préfiltrées (comme l'a souligné Marc Chénetier), et l'on peut alors observer l'image en train de se constituer et se figer. Les malentendus et chambres d'échos sont redoublés.

7 La conférence plénière de Marc Chénetier, «le passeur de la littérature américaine en France » (Sylvie Bauer), a été l'occasion d'une remise en question du terme de passeur contre le galvaudage contemporain du terme qui en émousse le sens. Mériter de se voir attribuer ce terme n'est pas évident, car surgissent les figures de tous ceux qui en fait de passeurs ne sont qu'autant d'entremetteurs, profiteurs et exploiteurs, profitant de "l'enflure des engouements inversement proportionnels à la solidité des jugements esthétiques ». Le fait d'« exoticiser» pour mieux vendre rejoint alors la tendance actuelle à la marchandisation de la littérature et, par extension, de toute la sphère sociale et culturelle. La liberté des éditeurs devient non existante, puisque selon les mots d'Hannah Arendt, ils sont confrontés à la « dégradante obligation d'être de son temps ", dans un cycle où les goûts du public et ce qu'on juge bon de lui proposer s'entraînent mutuellement. C'est la figure individuée et romantique du passeur qui 
parait alors trop dépassée pour correspondre à la réalité contemporaine. Le rôle du véritable "passeur", qui serait davantage un découvreur, serait de faire passer des ombres, les invisibles essentiels dont on ne parle pas, dans un dévouement absolu au littéraire et à la rencontre, au lieu de se contenter d'être un « strict importateur du déjà connu».

8 Le deuxième atelier, "Adaptations et réappropriations ", a permis de poursuivre une réflexion portant sur les modifications du texte et le passage d'un côté de l'Atlantique à l'autre de caractéristiques proprement « américaines » (ou du moins imaginées comme telles). Crystel Pinçonnat, de l'université Aix-Marseille, dans son analyse « Léo Malet ou le roman américain Made in France », a offert une vision du père du roman noir français comme "faussaire » d'une littérature américaine « à la française » à partir de plusieurs composantes : le personnage du privé, un emprunt direct au roman noir, que Malet transforme en journaliste; et la ville "américaine »; ce faisant, il adapte deux des "sèmes moteurs caractéristiques du genre ", l'enveloppe urbaine et le corps qui la traverse. Ces deux éléments, basés moins sur une réalité américaine que l'image que l'on s'en fait en France à l'époque, sont retravaillés suivant des techniques du mouvement surréaliste que Malet a fréquenté de près avant de basculer du côté du roman noir.

Hicham Mazouz, d'Emory University, a proposé une communication portant sur « Jean Genet and Richard Wright: Writing between Mediation and Intertextuality " qui permettait de voir les personnages marginaux de Jean Genet comme une force de contestation de l'hégémonie. En utilisant les textes de Frantz Fanon et de Richard Wright pour montrer la vulnérabilité et les dynamiques de domination à l'œuvre dans Notre Dame des Fleurs, Hicham Mazouz montre que Genet met en scène, en France, une expérience des corps marginaux subissant la violence comme technique d'invisibilisation et d'affirmation de la domination. L'existence du marginal est le problème : si un corps marginal devient visible, il s'expose à la sanction. Le trope du «Flying African » offrait une échappatoire radicale par la fiction.

10 Enfin, Julie Vatin-Corfdir, de l'université Paris Sorbonne, a présenté l'adaptation française des Sorcières de Salem d'Arthur Miller en 1957, dans le contexte du maccarthysme, réalisée par Raymond Rouleau, avec Simone Signoret et Yves Montand dans les rôles du couple Proctor, et s'est attelée à mettre en valeur ses partis pris dans leur contexte. L'adaptation du texte s'y est en effet faite en suivant une dynamique double: la domestication de l'étranger qui permet de rendre le texte acceptable, à travers une traduction qui opère des choix parfois radicaux, et le jeu sur la triple lisibilité intertextuelle des trois couples, les Proctor, les Rosenberg, et les MontandSignoret, qui avait permis à l'époque de vendre la pièce au public français comme une histoire d'engagement politique.

11 La première journée s'est conclue sur le troisième atelier portant sur les figures du poète. La communication de Mathieu Perrot, de Lafayette College, PA, avait pour titre "When Poetry Happens : la "poésie en action" dans l'œuvre de Jean-Jacques Lebel ». Lebel, un des premiers à avoir traduit Allen Ginsberg et d'autres poètes de la Beat Generation, considérait la poésie comme devant, en tant qu'événement, incarner une forme de soulèvement collectif. Préférant les affinités transnationales à celles régionales ou locales, Lebel incarne une problématisation de la figure du passeur comme celui qui, conscient des convergences, collectionne diverses pratiques pour 
créer une barricade contre l'art institutionnalisé, en ouvrant un passage, un espace, à l'espoir et à la bienveillance humaine.

12 Annick Ettlin, de l'université de Genève, a fait porter son attention sur la figure de Langston Hughes comme poète engagé, et sur la récupération de ce statut par les éditions Seghers pour faire de Hughes une figure universaliste, en aplanissant un engagement déjà jugé peu radical par des revues comme Présence africaine. Les positions politiques des auteurs américains paraissent ainsi être des points d'achoppement pour les passeurs français, qui ont du mal à transplanter d'un continent à l'autre les idées et leur expression dans un contexte différent teinté de visions étroites de ce qu'est la situation américaine, notamment en matière de relations entre communauté afroaméricaine et société d'après-guerre.

Charlotte Estrade, de l'université Paris-Nanterre, dans sa communication « American Modernist Poetry in Paris from the 1910s to the 1930s : Both to and away from Paris », a présenté les poètes américains vivant à Paris et fonctionnant comme passeurs de leurs propres œuvres et de celles de leurs contemporains. Leurs réseaux et communautés littéraires se formaient selon trois modalités différentes : les salons, l'idée d'écrire dans une langue étrangère, et les magazines. Paris était un épicentre des communautés littéraires : le salon de Gertrude Stein a permis des réunions jusqu'aux années 30, mais cette image de Stein comme passeuse a conduit à se souvenir d'elle comme d'une personnalité permettant la diffusion du travail des autres, plutôt que comme d'une écrivaine. Ezra Pound a tenté de rivaliser avec Stein en constituant son propre salon, fréquenté par des artistes tels que Cocteau, des dadaïstes et des surréalistes. Tandis que la majorité des poètes américains du haut modernisme étaient exilés à Paris, en Italie, et à Londres, Charlotte Estrade remarque qu'ils se tournaient néanmoins vers les ÉtatsUnis et souhaitaient la reconnaissance de leurs textes par un public américain.

La discussion s'est tournée vers les transformations dans l'action du passage du texte et la tentation d'établir un lien entre rupture de la forme poétique et position politique. D'après Mathieu Perrot, Lebel n'a pas changé la société française, mais a contribué à son échelle à « secouer » certains milieux. Charlotte Estrade ajoute qu'il est possible à travers ces poètes de voir comment une fois une certaine forme de poétique arrivée, le politique est réinjecté. On constate une différence entre vision rétrospective déterministe de certaines rencontres et contingence de l'activité sociable des poètes de l'époque, ainsi qu'entre les projections de l'image des poètes par eux-mêmes et leurs passeurs.

15 Le 15 mars débutait le quatrième atelier, portant sur les sociabilités. Il commençait par une communication d'Aliko Songolo, de l'université du Wisconsin-Madison, sur «Le Salon de Clamart : entre négrophilie et négritude ». Aliko Songolo s'est concentré sur les littératures des États-Unis noirs en rapport avec celle de la France noire, et sur le rôle de Paulette Nardal comme passeuse. Paulette Nardal a été elle-même oubliée ; on a avancé comme raisons de cet oubli l'absence d'écrits romanesques ou poétiques, d'engagement dans le marxisme, et sa fidélité aux idéaux de la République française, mais elle était surtout, d'après Aliko Songolo, femme et féministe avant la lettre. Au fil des années 20, son appartement de la banlieue de Clamart était le lieu, tous les dimanches après-midi, de discussions portant sur la littérature, la philosophie, la politique, et surtout l'image et la place des personnes noires dans la société occidentale. Elle fut également à l'origine de la Revue du monde noir, en 1931, une des premières à accorder une tribune aux noirs du monde entier. Son rôle a été reconnu par Senghor, 
mais la disparition de ses archives dans l'incendie de sa maison pose un réel problème lorsque l'on cherche à dévoiler l'ampleur de son influence. La figure de Paulette Nardal comme lieu de convergence transnational et son statut de femme noire permettent de problématiser la passeuse comme cruciale, omniprésente et invisibilisée.

16 Jamie Korsmo, de l'université de Georgia, a donné une communication sur une institution: «A Brief History Ex Libris: the founding of the American Library Association ». L'American Library est issue d'un réseau de bibliothèques destinées aux soldats américains pendant la Première Guerre mondiale et présent en différents lieux où les troupes étaient basées : Le Mans, Saint-Aignan, Saveney, etc. Jamie Korsmo a eu accès aux archives et aux photographies de l'époque. En 1920, ALA a fait don de fonds (en livres et financiers) pour la création de l'American Library in Paris; elle fut d'abord un monument à la mémoire d'Alan Seeger, fils du fondateur Charles Seeger et auteur du poème "I Have a Rendezvous with Death ». La bibliothèque a également fondé une revue littéraire, Ex Libris, en 1923 ; originellement censée faire connaitre la littérature américaine contemporaine en Europe, elle a servi de « lieu » de sociabilité et de contact pour les expatriés américains.

Giulia Napoleone, de l'université de Rome 3, a exploré la figure de « Nathalie Clifford Barney, médiatrice culturelle, traductrice et mécène ». Son salon du 20 rue Jacob se voulait un espace d'internationalisme, de dialogue et, le vendredi après-midi, un espace physique à cheval entre le public et le privé, transformé en lieu de l'esprit d'une époque, l'entre-deux-guerres. Nathalie Barney, consciente de son rôle de passeuse, présentait les uns aux autres les convives qui pouvaient s'entendre. Giulia Napoleone a établi une infographie montrant l'influence de Nathalie Barney sur des auteurs et autrices majeur.e.s de leur temps, tels qu'Ezra Pound, Rainer Maria Rilke, Djuna Barnes, etc. : à la fois muse et hôtesse d'un temple de l'amitié, elle incarne ce que Pascale Casanova disait de Paris, un «méridien de Greenwich » de la littérature, non pas tant espace physique que fonction.

18 Andrea Pitozzi, de l'université de Bergame, a conclu cet atelier par sa communication sur « le Paris d'Henry Miller », dans laquelle il opère un va-et-vient entre Miller dans Paris et Paris dans les romans de Miller pour poursuivre la réflexion sur les lieux infra et extratextuels du passage. Le Paris de Henry Miller est un Paris du désespoir, de l'ennui et du temps posthume, plongé dans un état perpétuel de semi-conscience, à la fois réel et esthétisé. Sa temporalité est "paradoxale et toujours ouverte ». Paris est également le lieu d'une forme de liberté et de relatif succès pour un auteur longtemps quasi inconnu outre-Atlantique: Henry Miller a vu ses premiers livres traduits en France dans les années 40 , et censurés aussitôt, tandis qu'un certain milieu culturel s'attache à le défendre (notamment Georges Bataille et Maurice Blanchot) et à affirmer que la violence et l'obscénité montrent une écriture d'une humanité totale qui se rebelle, d'un homme total qui se laisse aller à la présence du monde.

La discussion a permis d'insister sur l'existence de différents espaces qui ne se croisaient pas : les différents salons, les différentes visions de Paris. Les présentations sur le Salon de Clamart et celui de Nathalie Barney se répondent en cela même qu'elles montrent une ségrégation.

20 Dominique Jeannerod dans sa conférence plénière «Passeurs de récits: les revues policières américaines traduites en France (1950-1970) » a montré à travers une analyse quantitative que les revues policières telles que Mystère Magazine, Suspense, Minuit et Le Saint Détective Magazine (toutes traduites de revues policières américaines) de l'après- 
guerre permettent de savoir ce dont les amateurs de littérature policière se nourrissaient, à l'aide d'un moissonnage de données et d'une restitution sous forme quantitative (auteurs, autrices, prévalence de certaines revues, etc.). Le canon de ces revues différait certes du canon littéraire mais également $\mathrm{du}$ canon policier produit pour et par les collections sur lesquelles ces revues prenaient appui; ces revues utilisaient parfois des nouvelles d'auteurs canonisés afin de se légitimer (Mystère Magazine avait ainsi publié, entre autres, une nouvelle d'Apollinaire). Le déclin des collections dans les années 70 a coïncidé avec celui des revues, avec auparavant une dépolitisation et une épuration des contenus, toujours dans une visée de rejeter l'étiquette de littérature de divertissement. C'est en réaction à cette épuration que sera plus tard créé le néo-polar, qui repolitise le genre. C'est aussi l'occasion de dresser le portrait des hommes qui ont servi de passeurs derrière ces structures historiques: Maurice Renault, créateur entre autres de Mystère Magazine, et Igor Maslowski, le critique attitré de la revue. L'étude des revues permet de remettre des auteurs sur notre radar, en faisant une autre histoire du roman noir américain grâce à une autre histoire de l'Amérique.

Le cinquième et dernier atelier portait sur les presses, agences et imprimeurs.

22 André Derval, directeur des collections de l'Institut Mémoires de l'Édition Contemporaine à Caen, a dressé un portrait du fonds archivistique et bibliographique en retraçant ses origines. Créé en 1988, l'IMEC est une association visant à conserver les archives des maisons d'édition et permettre la consultation des documents, d'abord dans une salle de lecture à Paris ouverte en 1989, puis dans un bâtiment historique près de Caen, l'Abbaye d'Ardenne, dont la salle de lecture a été ouverte en octobre 2004 suite à l'obtention d'un label européen et la conclusion d'un accord tripartite avec le ministère de la Culture et le Conseil Régional de Basse Normandie. Aucun autre organisme au monde ne contient autant d'archives d'éditeurs : plus de $18 \mathrm{~km}$ linéaires d'archives dans l'abbaye, auxquels s'ajoutent $9 \mathrm{~km}$ de réserve extérieure.

La communication de Fiona McMahon, de l'université de Bourgogne, permet de poursuivre l'enquête sur les structures matérielles du passage de la littérature : « How to Be Modern : The Darantière Press and 1920s print culture in France ». Elle a rappelé l'importance du volume de Jean-Michel Rabaté sur Darantière: Maurice Darantière, les années 20. En tant qu'imprimeur, Maurice Darantière a dirigé son imprimerie à Dijon de 1917 à 1928 à la suite de son père, Victor ; grâce à son inscription dans le réseau local, il a pu remplir une fonction de mécène d'auteurs sans garantie aucune que leurs œuvres pourraient avoir un intérêt commercial. Ses liens avec Adrienne Monnier et la Maison des amis des livres lui ont permis de diminuer la distance séparant les écrivains anglophones des maisons d'édition; il s'est constitué comme imprimeur décidant de prendre en charge des projets extravagants en langue étrangère, comme l'exotisme du cercle des hauts modernistes, tout en étant loyal à sa coterie française (Adrienne Monnier, et Sylvia Beach, notamment) et en faisant partie d'une certaine frange marginale de la société. Sa collaboration avec Gertrude Stein s'est étendue sur de longues années.

24 Laurence Cossu-Beaumont, dans sa communication sur "L'agence littéraire William et Jenny Bradley, 1923-1982 ", est revenue vers de nombreux auteurs et autrices déjà mentionnés, puisqu'elle mettait en valeur le rôle du couple Bradley dans le passage de littérature américaine en France et de littérature française aux États-Unis. La longévité de leur agence fut telle qu'on ne peut citer tous ceux qui ont bénéficié de leur aide. 
Laurence Cossu-Beaumont a souligné le rôle d'accompagnateur de l'agent, qui facilite la vie de l'auteur de manières diverses, avant, pendant et après la production de l'ouvrage. Les réseaux du couple Bradley venaient également de leur présence dans les salons, et du caractère rhizomatique de leur position qui recoupait les trajectoires de nombreux passeurs déjà mentionnés : dans le salon de Barney, on trouvait notamment Rémi de Gourmont, traduit par William Barney ; Jenny Bradley tenait son propre salon les jeudis à son domicile, et était en contact avec Adrienne Monnier. La littérature que les Bradley ont portée était protéiforme, et leurs interlocuteurs incroyablement variés.

Enfin, Anne Cadin, de Sorbonne Université, a conclu l'atelier par une étude quantitative et qualitative sur «La presse et ses passeurs méconnus, intermédiaires indispensables à la circulation de la littérature américaine en France dans les années 40 ». Le « moment américain du roman français » est marqué par le passage à un public très vaste, mais également deux divisions : entre les auteurs favorisés par ce qu'on appelle le "prisme Coindreau » et les autres, et entre une littérature qu'on considère comme dégradante (point précurseur de la littérature américaine considérée comme culture de masse) et une littérature en voie de mise au canon littéraire. Dans la presse, des universitaires et des chroniqueurs et chroniqueuses (Raymond Las Vergnas, Thomas Narcejac) s'exprimèrent sur un panorama de la littérature américaine pendant la guerre et tentèrent de théoriser ce phénomène. Des voix s'efforcèrent alors de nuancer le débat pour éviter la politisation outrancière des questionnements liés à la littérature américaine en France.

La discussion s'est tournée vers la différence entre passeurs et polémistes : ces derniers, dans les articles de journaux, étalent des idées reçues au nom de combats idéologiques extra-littéraires entre France et États Unis. Les débats journalistiques ne portent pas alors tant sur une idée de la littérature américaine que sur l'effet qu'on s'imagine qu'elle aura sur une France elle aussi mythologisée. Ce phénomène existait auparavant, et se poursuit encore aujourd'hui.

Ces deux journées d'étude ont été l'occasion de balayer le champ pluridisciplinaire des passeurs d'Amérique, qui sont des figures aussi diverses que la littérature qu'ils essaient de faire connaître. A travers les passeurs, ce sont également les supports ou obstacles matériels ainsi que les mécanismes de production, de transmission, de diffusion, d'invisibilisation ou de mise en valeur, et de critique et canonisation de la littérature qui sont soulignés: presses, agents littéraires, revues, salons, lieux physiques, lieux de l'esprit et objets matériels sont des éléments qui sont souvent laissés de côté mais ont un grand pouvoir de détermination de ce qui est ou n'est pas donné à voir au public.

\section{INDEX}

Thèmes : Actualité de la recherche 
AUTEUR

CHRISTELLE CENTI

Université Rennes 2 